\title{
Perioperative albuminuria and clinical model to predict acute kidney injury in paediatric cardiac surgery
}

\author{
Arushi Nautiyal ${ }^{1}$. Sidharth Kumar Sethi ${ }^{2} \cdot$ Rajesh Sharma $^{3} \cdot$ Rupesh Raina $^{4}\left({ }^{\circ} \cdot\right.$ Abhishek Tibrewal $^{4} \cdot$ Romel Akole $^{3}$. \\ Aditi Gupta $^{5} \cdot$ Anil Bhan $^{6} \cdot$ Shyam Bihari Bansal ${ }^{1}$
}

Received: 12 April 2021 / Revised: 3 July 2021 / Accepted: 5 July 2021 / Published online: 20 September 2021

(c) IPNA 2021

\begin{abstract}
Background AKI is an important complication post cardiac surgery in children. An early diagnosis can help in mitigating complications and allow for prognostication. Urinary albumin:creatinine ratio (ACR) as a biomarker can provide a cheaper and more accessible AKI risk assessment and prediction. There is a paucity of paediatric literature regarding its utility.

Methods This was a prospective observational study, enrolling all children aged 1 month to 18 years, who underwent cardiac surgery, with use of cardiopulmonary bypass. Cohort was divided into groups $<2$ years and $\geq 2$ years for analyses to account for differences in physiological albumin excretion with age.

Results Of 143 children enrolled in the study, 36 developed AKI. In both age groups, the post-operative ACR was higher than pre-operative ACR among patients with and without AKI. In the group aged $\geq 2$ years, the highest first post-operative ACR tertile $(>75.8 \mathrm{mg} / \mathrm{g})$ predicted post-operative AKI after adjusting for clinical variables (adjusted RR, 11.71; 1.85-16.59). In the group aged $<2$ years, the highest first post-operative ACR tertile $(>141.3 \mathrm{mg} / \mathrm{g})$ predicted post-operative AKI in unadjusted analysis but not after adjusting for clinical variables (RR, 2.78; 0.70-6.65). For AKI risk prediction, AUC (95\% CI) was highest after combining clinical model and pre-operative ACR for groups aged $<2$ years $[0.805(0.713-0.896)]$ and $\geq 2$ years $[0.872(0.772-0.973)]$.

Conclusions This study provides evidence for use of albuminuria as a feasible biomarker in AKI prediction in children post cardiac surgery, especially when added to a clinical model.
\end{abstract}

Keywords Acute kidney injury $\cdot$ Cardiac surgery $\cdot$ Cardiopulmonary bypass $\cdot$ Proteinuria $\cdot$ Albuminuria $\cdot$ Risk prediction

Both Dr. Arushi Nautiyal and Dr. Sidharth Kumar Sethi contributed equally and shall be first authors.

Rupesh Raina

rraina@akronchildrens.org

1 Kidney Institute, Medanta, The Medicity Hospital, Haryana, Gurgaon 122001, India

2 Pediatric Nephrology, Kidney Institute, Medanta, The Medicity Hospital, Haryana, Gurgaon 122001, India

3 Pediatric Cardiac Intensive Care, Medanta, The Medicity Hospital, Haryana, Gurgaon 122001, India

4 Akron's Children Hospital, Akron, OH, USA

5 Aster Clinical Lab, Bangalore, India

6 CTVS, Medanta, The Medicity Hospital, Haryana, Gurgaon 122001, India

\section{Introduction}

Acute kidney injury (AKI) in children following cardiac surgery is common and associated with poor outcomes; it leads to increase in hospital stay, need for ventilator support, escalation of costs and even mortality [1-4]. The rise of serum creatinine is widely used for defining AKI; however, it often rises after significant kidney function has already been lost [5]. Biomarkers have shown utility with regard to a more expeditious diagnosis of AKI and prognostication of AKI as well as application of preventive strategies [5]. As previously studied, the use of biomarkers helped in enhancing the effectiveness of renal angina index (RAI) in AKI risk stratification of critically ill patients [6]. Similarly, a randomised trial in cardiac surgery patients was able to significantly reduce AKI by applying a care bundle in patients at high risk of kidney dysfunction, identified by the use of urinary biomarkers [7]. However, the applicability of most serum 
or urinary biomarkers is limited due to issues of cost, standardisation and lack of generalizability and being not yet in mainstream use. Urinary biomarker albumin:creatinine ratio (ACR) on the other hand, which is frequently used in assessment of chronic kidney disease, could provide a cheaper, more familiar and accessible option for AKI assessment [8].

As normal glomerular function limits albumin filtration and renal tubules are responsible for albumin reclamation, albuminuria can thus develop in AKI following ischemic or inflammatory insults to these structures $[9,10]$. Various animal and human clinical studies have identified albuminuria developing in AKI states [9-11]. Studies in adults have identified pre-existing proteinuria as a risk factor for AKI in cardiac surgery $[12,13]$. There have been limited studies in the paediatric population assessing albuminuria in AKI [14]. This study was designed to assess pre- and post-operative albuminuria in children undergoing cardiac surgery with cardiopulmonary bypass and its ability to predict AKI as described by KDIGO criteria [15].

\section{Methods}

\section{Aim}

To assess albuminuria as a predictive marker for AKI in children (1 month-18 years) undergoing cardiac surgery using cardiopulmonary bypass (open cardiac surgery) in both preand post-operative periods.

\section{Study design}

This was a prospective, observational study. Children undergoing cardiac surgery with use of cardiopulmonary bypass at our Paediatric Cardiothoracic unit from October 2017 to December 2018 were enrolled in this study.

\section{Inclusion criteria}

Children between 1 month and 18 years undergoing elective cardiac surgery were included.

\section{Exclusion criteria}

Children with pre-existing kidney dysfunction (baseline eGFR $<60 \mathrm{~mL} / \mathrm{min} / 1.73 \mathrm{~m}^{2}$, structural kidney abnormalities) or post kidney transplant were excluded, as well as those with incomplete data.

Informed consent was taken from all parents prior to the inclusion in the study and assent where applicable. This study was approved by the Research Ethics Board of the institution.

\section{Collection of data}

All children included in the study were admitted for open cardiac surgery at our cardiothoracic unit. Demographic variables including age, gender, weight and length were recorded prior to operative procedure. Data pertaining to diagnosis of heart disease, acyanotic/cyanotic heart disease and prior surgeries was collected. Glomerular filtration rate was estimated using the revised bedside Schwartz formula [16]. Urine ACR was measured in the pre-operative period. Urine was collected via Foley's catheter and using a clean catch sample in those without urinary catheter.

Intraoperative variables such as duration of procedure, length of cardiopulmonary bypass and aortic cross clamp times were recorded. RACHS-1 category was assigned to each child; it is a consensus-based tool which defines short-term mortality risk in children undergoing surgery for congenital heart disease based on the type of procedure performed [17]. Post-operatively serum creatinine was measured daily until ICU stay and 1 day prior to hospital discharge. AKI was defined as per KDIGO definitions [15]. AKI stage 1 was defined as increase of serum creatinine $\geq 0.3 \mathrm{mg} / \mathrm{dL}$ within $48 \mathrm{~h}$ or $\geq 1.5$ - to two-fold from baseline or urine output $\leq 0.5 \mathrm{~mL} / \mathrm{kg} / \mathrm{h}$ for $6-12 \mathrm{~h}$. AKI stage 2 was defined as a rise of serum creatinine 2.0-2.9 times from baseline or urine output $\leq 0.5 \mathrm{~mL} / \mathrm{kg} / \mathrm{h}$ for $12 \mathrm{~h}$. AKI stage 3 was defined as a rise of serum creatinine 3.0 times from baseline or a rise to $\geq 4.0 \mathrm{mg} / \mathrm{dL}$ or initiation of kidney replacement therapy or, in patients $<18$ years, decrease in eGFR to $<35 \mathrm{~mL} / \mathrm{min}$ per $1.73 \mathrm{~m}^{2}$ or urine

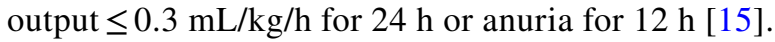

Daily and cumulative fluid balances were recorded, as per the method used by Goldstein et al. [18]. Urine ACR was measured $6 \mathrm{~h}$ post-operatively on day 0 , followed by daily for the next 2 days. Data pertaining to duration of mechanical ventilator support, use of NSAIDs/nephrotoxic drugs, duration of hospital stay, development of sepsis, transaminitis (more than two times the upper limit of normal), in-hospital mortality and development of low output cardiac syndrome (symptoms and signs of poor systemic perfusion such as tachycardia, cold extremities or development of acidosis such as increase in base deficit $>4$ or increase in lactate by $>2 \mathrm{mg} / \mathrm{dL}$ in consecutive blood gas analysis; criteria used by Hoffman et al. [19]) were recorded. Haematological complications referred to development of anaemia and/or thrombocytopenia during admission. Diuretics and kidney replacement therapy were provided where required. Urine ACR was measured by an immunoturbidimetry method using the Architect ci-4100 analyser. Urine creatinine was measured by enzymatic method (creatinine amidohydrolase). All the analyses 
were conducted separately for children aged $<2$ years and $\geq 2$ years, as younger children tend to have physiological higher urinary albumin excretion which stabilises with tubular function maturation [14]. For the same reason, due to the variability in protein and creatinine excretion among neonates, especially early after birth, it was considered to exclude this age group from the analysis, as the physiological variations may become a confounding factor in evaluation.

\section{Statistical analyses}

The variables were recorded in Microsoft Excel and statistical software (SPSS version 22) was used for the statistical analyses. All the variables were tested for normality using the Kolmogorov-Smirnov test. The outcomes include AKI incidence (yes vs. no, and stage $\geq 2$ vs. stage 1 ), length of stay (ICU and hospital) and mortality. The categorical variables were summarized as frequencies and percentages, while continuous variables as median and interquartile range (IQR; 25th to 75th percentiles) for the sake of consistency. The association between categorical variables was assessed using the chi-square statistic or Fischer exact test, while continuous variables with the Mann-Whitney $U$ test or Kruskal-Wallis test. Generalized linear model was used to calculate the unadjusted and adjusted relative risks (RRs) of pre-operative and post-operative day 0 (first post-operative) ACR for the prediction of AKI. The pre-operative ACR was categorized as ACR $<10 \mathrm{mg} / \mathrm{g}$, ACR $10-30 \mathrm{mg} / \mathrm{g}$ and ACR $>30 \mathrm{mg} / \mathrm{g}$, while the first post-operative ACR was categorized based on its tertile values.

The multivariate clinical model comprised multiple predictors, such as age (continuous in month for the group aged $<2$ years; categorical for the group aged $\geq 2$ years: 2 $-<6$ vs. $\geq 6$ years); gender (categorical: male vs. female); previous cardiac surgeries (categorical: yes vs. no); type of heart surgery (categorical: cyanotic vs. acyanotic); RACHS-1 score (categorical: $\geq 3$ vs. $<3$ ); cardiopulmonary bypass time (categorical: $<120 \mathrm{vs} . \geq 120 \mathrm{~min}$ ); pre-operative creatinine (continuous in $\mathrm{mg} / \mathrm{dL}$ ) and pre-operative estimated glomerular filtration rate (eGFR; continuous in $\mathrm{mL} /$ $\min / 1.73 \mathrm{~m}^{2}$ ).

The different diagnostic characteristics (sensitivity, specificity, positive predictive value and negative predictive value) of pre-operative and first post-operative ACR threshold values for AKI prediction were conducted. The area under the receiver operating characteristic curve (AUC; with $95 \%$ confidence interval $(95 \% \mathrm{CI})$ ) was calculated to determine the ability of clinical model alone, pre-operative ACR alone, first post-operative ACR alone and combinations of these to discriminate between patients with and without AKI. A two tailed $p$ value $<0.05$ was considered to be statistically significant.

\section{Results}

\section{Patient characteristics}

The study flow is shown in Supplementary Fig. 1. In the group aged $<2$ years, the median (IQR) age was significantly lower among those with higher ACR level (ACR < $10 \mathrm{mg} / \mathrm{g}: 12$ (-) months vs. ACR $10-30 \mathrm{mg} / \mathrm{g}: 9$ (7.3-12) months vs. ACR $>30 \mathrm{mg} / \mathrm{g}: 6$ (4-12) months; $p<0.05)$ and the proportion of AKI incidence was significantly higher among those with higher ACR level (ACR $<10 \mathrm{mg} / \mathrm{g}: 0 \%$ vs. ACR $10-30 \mathrm{mg} / \mathrm{g}: 5 \%$ vs. ACR $>30 \mathrm{mg} / \mathrm{g}: 35 \% ; p<0.05)$. All other variables did not differ significantly across the three pre-operative ACR levels. Similarly, among the group aged $\geq 2$ years, none of the variables differed significantly across the three pre-operative ACR levels (Table 1). Out of 143 children, 93 (65\%) were aged $<2$ years and $50(35 \%)$ were aged $\geq 2$ years. Thirty-six children developed AKI; 26 were $<2$ years and 10 were $\geq 2$ years of age. Four children required peritoneal dialysis. AKI occurred first on mean POD 1.96 (SD 1.34 ) in children $<2$ years and on mean POD 1.5 in children $\geq 2$ years (SD 0.97).

\section{Association of pre-operative and post-operative ACR with AKI}

In both age groups, the post-operative ACR was much higher than pre-operative ACR among patients with and without AKI. A fall in urine ACR was also seen in all age groups by POD2. In the group aged $<2$ years, the median (IQR) ACR was significantly higher among those with AKI as compared to those without AKI: at pre-operative $(64.5(41-86.8)$ vs. $40(25-55) \mathrm{mg} / \mathrm{g} ; p<0.05)$, at postoperative day 0 (153 (98-347.8) vs. $99(54-142) \mathrm{mg} / \mathrm{g}$; $p<0.05)$, at post-operative day $1(283.5(127-455)$ vs. $97(68-164) \mathrm{mg} / \mathrm{g} ; p<0.001)$ and at post-operative day 2 (197.5 (89-355) vs. $74(53-122) \mathrm{mg} / \mathrm{g} ; p<0.001)$. However, in the group aged $\geq 2$ years, the median (IQR) was significantly higher among those with AKI as compared to those without AKI only at post-operative day 0 (131.5 (78-362.5) vs. 48 (22-75) mg/g; $p<0.05$ ) (Table 2).

In the group aged $<2$ years, the pre-operative ACR $>30 \mathrm{mg} / \mathrm{g}$ predicted post-operative AKI in unadjusted analysis (RR 7.29; 95\% CI, 1.05-50.68) but not after adjusting for clinical variables (RR 4.91; 0.7-15.33) (Table 3 ). In the group aged $\geq 2$ years, the pre-operative ACR $>30 \mathrm{mg} / \mathrm{g}$ was not significantly associated with postoperative AKI. Also, in the group aged $<2$ years, preoperative ACR $>30 \mathrm{mg} / \mathrm{g}$ was not significantly associated with the development of stage $\geq 2$ AKI. Meanwhile, in the 
Table 1 Patient characteristics by age group and by pre-operative ACR category

\begin{tabular}{|c|c|c|c|c|c|c|}
\hline \multirow[t]{2}{*}{ Variable } & \multicolumn{3}{|l|}{$<2$ years } & \multicolumn{3}{|l|}{$\geq 2$ years } \\
\hline & $\begin{array}{l}\mathrm{ACR}<10 \mathrm{mg} / \mathrm{g} \\
(n=1)\end{array}$ & $\begin{array}{l}\text { ACR } 10-30 \mathrm{mg} / \mathrm{g} \\
(n=20)\end{array}$ & $\begin{array}{l}\text { ACR }>30 \mathrm{mg} / \mathrm{g} \\
(n=72)\end{array}$ & $\begin{array}{l}\mathrm{ACR}<10 \mathrm{mg} / \mathrm{g} \\
(n=6)\end{array}$ & $\begin{array}{l}\text { ACR } 10-30 \mathrm{mg} / \mathrm{g} \\
(n=37)\end{array}$ & $\begin{array}{l}\mathrm{ACR}>30 \mathrm{mg} / \mathrm{g} \\
(n=7)\end{array}$ \\
\hline \multicolumn{7}{|l|}{$\begin{array}{l}\text { Continuous variables } \\
\text { [median (IQR)] }\end{array}$} \\
\hline Age (months) & $12(-)$ & $9(7.3-12)$ & $6(4-12)^{\mathrm{a}}$ & $66(45-132)$ & $60(36-96)$ & $132(48-168)$ \\
\hline $\begin{array}{l}\text { Pre-operative cre- } \\
\text { atinine (mg/dL) }\end{array}$ & $0.3(-)$ & $0.3(0.3-0.4)$ & $0.3(0.3-0.3)$ & $0.4(0.4-0.5)$ & $0.4(0.3-0.4)$ & $0.4(0.3-0.5)$ \\
\hline $\begin{array}{l}\text { Pre-operative GFR } \\
(\mathrm{mL} / \mathrm{min} / 1.73 \\
\left.\mathrm{m}^{2}\right)\end{array}$ & $103(-)$ & $88.5(76.3-108.8)$ & $85(74-103)$ & $99(95.3-115.5)$ & $116(98-134)$ & $109(99-126)$ \\
\hline $\begin{array}{l}\text { Duration of opera- } \\
\text { tion (h) }\end{array}$ & $4(-)$ & $4.5(4-5)$ & $5(4-5.8)$ & $4.5(2.5-5.3)$ & $5(4-5)$ & $6(5-6)$ \\
\hline CPB time (min) & $66(-)$ & $64(48.5-71.5)$ & $61(50.3-79)$ & $52(30.5-65.5)$ & $57(45-74)$ & $58(48-99)$ \\
\hline $\begin{array}{l}\text { Cumulative fluid } \\
\text { overload (\%) }\end{array}$ & $-15 \%(-)$ & $-16 \%(-21$ to $-8 \%)$ & $-19 \%(-27$ to $-12 \%)$ & $-5 \%(-11$ to $-2 \%)$ & $-9 \%(-14$ to $-6 \%)$ & $\begin{array}{c}-10 \%(-18 \\
\text { to }-5 \%)\end{array}$ \\
\hline \multicolumn{7}{|l|}{$\begin{array}{l}\text { Categorical variables } \\
{[n(\%)]}\end{array}$} \\
\hline Gender (male) & $0(0 \%)$ & $16(80 \%)$ & $47(65.3 \%)$ & $2(33.3 \%)$ & $24(64.9 \%)$ & $5(71.4 \%)$ \\
\hline $\begin{array}{l}\text { Type of heart dis- } \\
\text { ease (cyanotic) }\end{array}$ & $0(0 \%)$ & $7(35 \%)$ & $40(55.6 \%)$ & $3(50 \%)$ & $18(48.6 \%)$ & $4(57.1 \%)$ \\
\hline $\begin{array}{r}\text { Previous cardiac } \\
\text { surgeries (yes) }\end{array}$ & $0(0 \%)$ & $1(5 \%)$ & $1(1.4 \%)$ & $1(16.7 \%)$ & $10(27 \%)$ & $1(14.3 \%)$ \\
\hline RACHS-1 score & $2(-)$ & $2(2-2.8)$ & $2(2-3)$ & $2(1-2)$ & $2(2-3)$ & $2(2-3)$ \\
\hline $\begin{array}{l}\text { ACC time } \\
\quad(<60 \mathrm{~min})\end{array}$ & $1(100 \%)$ & $16(80 \%)$ & $53(74.6 \%)$ & $5(100 \%)$ & $26(81.2 \%)$ & $5(71.4 \%)$ \\
\hline Sepsis (yes) & $0(0 \%)$ & $6(30 \%)$ & $34(47.2 \%)$ & $0(0 \%)$ & $11(29.7 \%)$ & $0(0 \%)$ \\
\hline $\begin{array}{l}\text { Haematological } \\
\text { complications } \\
\text { (yes) }\end{array}$ & $1(100 \%)$ & $4(20 \%)$ & $17(23.6 \%)$ & $1(16.7 \%)$ & $1(2.7 \%)$ & $2(28.6 \%)$ \\
\hline Transaminitis (yes) & $0(0 \%)$ & $9(45 \%)$ & $46(63.9 \%)$ & $1(16.7 \%)$ & $13(35.1 \%)$ & $4(57.1 \%)$ \\
\hline $\begin{array}{l}\text { Low cardiac output } \\
\text { syndrome (yes) }\end{array}$ & $0(0 \%)$ & $8(40 \%)$ & $42(58.3 \%)$ & $0(0 \%)$ & $17(45.9 \%)$ & $4(57.1 \%)$ \\
\hline AKI (yes) & $0(0 \%)$ & $1(5 \%)$ & $25(34.7 \%)^{\mathrm{a}}$ & $0(0 \%)$ & $8(21.6 \%)$ & $2(28.6 \%)$ \\
\hline AKI staging $(\geq 2)$ & $0(0 \%)$ & $1(100 \%)$ & $14(56 \%)$ & $0(0 \%)$ & $2(25 \%)$ & $0(0 \%)$ \\
\hline AKI recovery (yes) & $0(0 \%)$ & $0(0 \%)$ & $24(96 \%)$ & $0(0 \%)$ & $7(87.5 \%)$ & $2(100 \%)$ \\
\hline $\begin{array}{l}\text { Need for KRT } \\
\quad \text { (yes) }\end{array}$ & $0(0 \%)$ & $1(100 \%)$ & $2(8 \%)$ & $0(0 \%)$ & $1(12.5 \%)$ & $0(0 \%)$ \\
\hline $\begin{array}{l}\text { Re-explora- } \\
\text { tion / 2nd opera- } \\
\text { tion (yes) }\end{array}$ & $0(0 \%)$ & $1(5 \%)$ & $2(2.8 \%)$ & $0(0 \%)$ & $2(5.4 \%)$ & $0(0 \%)$ \\
\hline $\begin{array}{l}\text { Prolonged ventila- } \\
\text { tion (yes) }\end{array}$ & $1(100 \%)$ & $19(95 \%)$ & $56(77.8 \%)$ & $6(100 \%)$ & $33(89.2 \%)$ & $5(71.4 \%)$ \\
\hline Re-intubation (yes) & $0(0 \%)$ & $1(5 \%)$ & $10(13.9 \%)$ & $0(0 \%)$ & $1(2.7 \%)$ & $0(0 \%)$ \\
\hline $\begin{array}{l}\text { Nephrotoxic antibi- } \\
\text { otics used (yes) }\end{array}$ & $1(100 \%)$ & $17(85 \%)$ & $55(76.4 \%)$ & $6(100 \%)$ & $29(78.4 \%)$ & $5(71.4 \%)$ \\
\hline Death (yes) & $0(0 \%)$ & $1(5 \%)$ & $1(1.4 \%)$ & $0(0 \%)$ & $1(2.7 \%)$ & $0(0 \%)$ \\
\hline
\end{tabular}

$G F R$, glomerular filtration rate; $m g$, milligram; $d L$, decilitre; $m L$, millilitre; $m i n$, minute; $m$, metre; $R A C H S$, risk adjustment in congenital heart surgery; $C P B$, cardiopulmonary bypass; $A K I$, acute kidney injury; $K R T$, kidney replacement therapy; $A C C$, aortic cross clamp; $g$, gram; $A C R$, albumin-to-creatinine ratio; $I Q R$, interquartile range

${ }^{\mathrm{a}} p<0.05$

group aged $\geq 2$ years, it was not possible to calculate the risk for pre-operative ACR to predict stage $\geq 2$ AKI due to low event numbers (Table 3).

In the group aged $\geq 2$ years, the highest first post-operative ACR tertile $(>75.8 \mathrm{mg} / \mathrm{g})$ predicted post-operative
AKI after adjusting for clinical variables (unadjusted RR, 8; 1.48-15.13; adjusted RR 11.71; 1.85-16.59) (Table 4). In the group aged $<2$ years, the highest first post-operative ACR tertile ( $>141.3 \mathrm{mg} / \mathrm{g}$ ) predicted post-operative AKI in unadjusted analysis (RR 4.52; 1.71-7.67) but not 
Table 2 Comparison of preoperative and post-operative ACR among children with and without AKI, by age group

\begin{tabular}{lccccr}
\hline ACR & \multicolumn{2}{c}{$<$ years [median $(\mathrm{IQR})] \mathrm{mg} / \mathrm{g}$} & & \multicolumn{2}{c}{$\geq 2$ years [median $(\mathrm{IQR})] \mathrm{mg} / \mathrm{g}$} \\
\cline { 2 - 3 } & Yes $(n=26)$ & No $(n=67)$ & & Yes $(n=10)$ & No $(n=50)$ \\
\hline Pre-operative & $64.5(41-86.8)$ & $40(25-55)^{\mathrm{a}}$ & & $17.5(14.3-31.5)$ & $15(11.25-20)$ \\
POD day 0 & $153(98-347.8)$ & $99(54-142)^{\mathrm{a}}$ & & $131.5(78-362.5)$ & $48(22-75)^{\mathrm{a}}$ \\
POD day 1 & $283.5(127-455)$ & $97(68-164)^{\mathrm{b}}$ & & $189.5(29.3-396)$ & $47.5(23.3-82.3)$ \\
POD day 2 & $197.5(89-355)$ & $74(53-122)^{\mathrm{b}}$ & & $170(25-241)$ & $30(18.3-73.3)$ \\
\hline
\end{tabular}

$P O D$, post-operative day; $A C R$, albumin-to-creatinine ratio

${ }^{\mathrm{a}} p<0.05 ;{ }^{\mathrm{b}} p<0.001$

Table 3 Unadjusted and adjusted RRs for pre-operative ACR to predict post-operative AKI or stage $\geq 2$ AKI, by age group

\begin{tabular}{|c|c|c|c|c|c|c|}
\hline Pre-operative ACR & AKI (yes vs. no) & $\begin{array}{l}\text { Unadjusted RR } \\
(95 \% \mathrm{CI})\end{array}$ & $\begin{array}{l}\text { Adjusted RR (95\% } \\
\text { CI) }\end{array}$ & AKI $\geq$ stage 2 & $\begin{array}{l}\text { Unadjusted RR } \\
(95 \% \mathrm{CI})\end{array}$ & $\begin{array}{l}\text { Adjusted RR (95\% } \\
\text { CI) }\end{array}$ \\
\hline \multicolumn{7}{|l|}{$<2$ years $^{b}$} \\
\hline No. of events & 26 & & & 15 & & \\
\hline $\begin{array}{c}\mathrm{ACR} \leq 30 \mathrm{mg} / \mathrm{g} \\
(n=21)\end{array}$ & $1(4.8 \%)$ & 1 & 1 & $1(4.8 \%)$ & 1 & 1 \\
\hline $\begin{array}{l}\text { ACR }>30 \mathrm{mg} / \mathrm{g} \\
\quad(n=72)\end{array}$ & $25(34.7 \%)$ & $7.29(1.05-50.68)$ & $4.91(0.7-15.33)$ & $14(19.4 \%)$ & $4.08(0.57-29.27)$ & $2.03(0.22-11)$ \\
\hline \multicolumn{7}{|l|}{$\geq 2$ years $^{c}$} \\
\hline No. of events & 10 & & & 2 & & \\
\hline $\begin{array}{l}\mathrm{ACR} \leq 30 \mathrm{mg} / \mathrm{g} \\
(n=43)\end{array}$ & $8(18.6 \%)$ & 1 & 1 & $2(4.7 \%)$ & 1 & 1 \\
\hline $\begin{array}{l}\text { ACR }>30 \mathrm{mg} / \mathrm{g} \\
\quad(n=7)\end{array}$ & $2(28.6 \%)$ & $1.54(0.41-5.79)$ & $1.71(0.31-4.2)$ & $0(0 \%)$ & NA & NA \\
\hline
\end{tabular}

$R R$, relative risk; $A C R$, albumin-to-creatinine ratio; $A K I$, acute kidney injury; $G F R$, glomerular filtration rate; $R A C H S$, risk adjustment in congenital heart surgery; $N A$, not applicable

${ }^{\mathrm{b}}$ Adjusted for age (continuous), gender (categorical), creatinine (continuous), GFR (continuous), type of heart surgery (categorical), previous cardiac surgeries (categorical) and RACHS- 1 score $\geq 3$ versus $<3$ (categorical)

${ }^{\mathrm{c}}$ Adjusted for age (categorical; $<6$ and $\geq 6$ years), gender (categorical), creatinine (continuous), GFR (continuous), type of heart surgery (categorical), previous cardiac surgeries (categorical) and RACHS-1 score $\geq 3$ versus $<3$ (categorical)

after adjusting for clinical variables (RR 2.78; 0.70-6.65) (Table 4). However, in the group aged $<2$ years, the first post-operative ACR tertiles were not significantly associated with the development of stage $\geq 2$ AKI. Meanwhile, in the group aged $\geq 2$ years, it was not possible to calculate the risk for post-operative ACR to predict stage $\geq 2$ AKI due to low event numbers (Table 4).

\section{Association of pre- and post-operative ACR with duration of stay and mortality}

In the group aged $<2$ years, the median duration of ICU stay and duration of hospital stay increased significantly with advancing pre-operative $(p<0.05)$, post-operative day $0(p<0.05)$, post-operative day $1(p<0.001)$ and post-operative day $2(p<0.05)$ ACR levels (Table 5). Similarly, in the group aged $\geq 2$ years, the median duration of ICU stay and duration of hospital stay increased significantly with advancing post-operative day $0(p<0.05)$, post-operative day $1(p<0.05)$ and post-operative day $2(p<0.001)$ ACR levels, but were not significantly associated with pre-operative ACR levels (Table 5). There were two deaths in the group aged $<2$ years, the median (IQR) ACR level at postoperative day 2 was significantly higher among those who died as compared to that among those who did not die (419 $(298-540)$ vs. $82(58-180) \mathrm{mg} / \mathrm{g} ; p<0.05)$. Also, median (IQR) ACR levels at different time points were higher among those who died vs. did not die; however, there was a lack of statistical significance, probably due to limited sample size in the mortality group. There was one death in the age group $\geq 2$ years: the highest ACR was observed on POD1, which was higher than those who did not die (350 vs. median IQR 61 (25-90.5) mg/g).

Table 6 displays the diagnostic characteristics of preoperative and first post-operative ACR thresholds for AKI diagnosis. For the pre-operative ACR and first post-operative ACR, the ACR threshold value with maximal sensitivity and specificity in the group aged $\geq 2$ years was almost one-third and three-fifths of the similar value in the group aged $<2$ years, respectively. In general, negative predictive 
Table 4 Unadjusted and adjusted RRs for first post-operative ACR to predict post-operative AKI or stage $\geq 2$ AKI, by age group

\begin{tabular}{|c|c|c|c|c|c|c|}
\hline $\begin{array}{l}\text { 1st post-operative } \\
\text { ACR }\end{array}$ & AKI (yes vs. no) & $\begin{array}{l}\text { Unadjusted RR } \\
(95 \% \mathrm{CI})\end{array}$ & $\begin{array}{l}\text { Adjusted RR (95\% } \\
\text { CI) }\end{array}$ & AKI $\geq$ stage 2 & $\begin{array}{l}\text { Unadjusted RR } \\
\text { (95\% CI) }\end{array}$ & $\begin{array}{l}\text { Adjusted RR (95\% } \\
\text { CI) }\end{array}$ \\
\hline \multicolumn{7}{|l|}{$<2$ years $^{\mathrm{b}}$} \\
\hline No. of events & 26 & & & 15 & & \\
\hline $\begin{array}{l}\text { 1st tertile } \\
\qquad \begin{array}{l}(<75 \mathrm{mg} / \mathrm{g} ; \\
n=30)\end{array}\end{array}$ & $3(10.0 \%)$ & 1 & 1 & $2(6.7 \%)$ & 1 & 1 \\
\hline $\begin{array}{l}\text { 2nd tertile (75- } \\
\qquad \begin{array}{l}141.3 \mathrm{mg} / \mathrm{g} \\
n=32)\end{array}\end{array}$ & $9(28.1 \%)$ & $2.81(0.86-6.18)$ & $2.60(0.70-6.23)$ & $5(15.6 \%)$ & $2.34(0.48-7.64)$ & $1.78(0.30-7.12)$ \\
\hline $\begin{array}{l}\text { 3rd tertile } \\
\qquad \begin{array}{l}>141.3 \mathrm{mg} / \mathrm{g} \\
n=31)\end{array}\end{array}$ & $14(45.2 \%)$ & $4.52(1.71-7.67)$ & $2.78(0.70-6.65)$ & $8(25.8 \%)$ & $3.87(0.94-9.65)$ & $1.47(0.23-6.52)$ \\
\hline \multicolumn{7}{|l|}{$\geq 2$ years $^{c}$} \\
\hline No. of events & 10 & & & 2 & & \\
\hline $\begin{array}{l}\text { 1st tertile } \\
\qquad \begin{array}{l}(<35.9 \mathrm{mg} / \mathrm{g} ; \\
n=17)\end{array}\end{array}$ & $1(5.9 \%)$ & 1 & 1 & $0(0 \%)$ & 1 & 1 \\
\hline $\begin{array}{l}\text { 2nd tertile (35.9- } \\
\begin{array}{l}75.8 \mathrm{mg} / \mathrm{g} \\
n=16)\end{array}\end{array}$ & $1(6.25 \%)$ & $1.06(0.07-9.13)$ & $1.10(0.04-11.71)$ & $0(0 \%)$ & NA & NA \\
\hline $\begin{array}{l}\text { 3rd tertile } \\
\qquad \begin{array}{l}(>75.8 \mathrm{mg} / \mathrm{g} ; \\
n=17)\end{array}\end{array}$ & $8(47.1 \%)$ & $8(1.48-15.13)$ & $11.71(1.85-16.59)$ & $2(11.8 \%)$ & NA & NA \\
\hline
\end{tabular}

$R R$, relative risk; $A C R$, albumin-to-creatinine ratio; $A K I$, acute kidney injury; $G F R$, glomerular filtration rate; $R A C H S$, risk adjustment in congenital heart surgery; $N A$, not applicable

${ }^{\mathrm{b}}$ Adjusted for age (continuous), gender (categorical), creatinine (continuous), GFR (continuous), type of heart surgery (categorical), previous cardiac surgeries (categorical) and RACHS-1 score $\geq 3$ versus $<3$ (categorical)

${ }^{\mathrm{c}}$ Adjusted for age (categorical; $<6$ and $\geq 6$ years), gender (categorical), creatinine (continuous), GFR (continuous), type of heart surgery (categorical), previous cardiac surgeries (categorical) and RACHS-1 score $\geq 3$ versus $<3$ (categorical)

Table 5 Comparison of duration of ICU stay and hospital stay by pre-operative and post-operative ACR levels, by age groups

\begin{tabular}{llll}
\hline ACR & & $\begin{array}{l}\text { Duration of ICU stay (days) } \\
\text { Median }(\mathrm{IQR}) \mathrm{mg} / \mathrm{g}\end{array}$ & $\begin{array}{l}\text { Duration of hospital stay (days) } \\
\text { Median }(\mathrm{IQR}) \mathrm{mg} / \mathrm{g}\end{array}$ \\
\hline$<$ 2 years & & & $6(-)$ \\
Pre-operative & $<10 \mathrm{mg} / \mathrm{g}(n=1)$ & $3(-)$ & $8(7-10.5)$ \\
& $10-30 \mathrm{mg} / \mathrm{g}(n=20)$ & $4(3-4.75)$ & $10(8-14.75)^{\mathrm{a}}$ \\
& $>30 \mathrm{mg} / \mathrm{g}(n=72)$ & $5(4-7.75)^{\mathrm{a}}$ & $12(8-19)^{\mathrm{a}}$ \\
POD day 0 & 3rd tertile $(>141.3 \mathrm{mg} / \mathrm{g} ; n=31)$ & $6(4-10)^{\mathrm{a}}$ & $12(8-19)^{\mathrm{b}}$ \\
POD day 1 & 3rd tertile $(>176.9 \mathrm{mg} / \mathrm{g} ; n=31)$ & $6(4-12)^{\mathrm{b}}$ & $12(8-17)^{\mathrm{a}}$ \\
POD day 2 & 3rd tertile $(>135.7 \mathrm{mg} / \mathrm{g} ; n=31)$ & $6(4-12)^{\mathrm{a}}$ & $6.5(5-7.75)$ \\
$\geq 2$ years & & & $8(6.5-9)$ \\
Pre-operative & $<10 \mathrm{mg} / \mathrm{g}(n=6)$ & $3(2.75-3.75)$ & $9(6-12)$ \\
& $10-30 \mathrm{mg} / \mathrm{g}(n=37)$ & $3(3-4.5)$ & $9(7.5-11)^{\mathrm{a}}$ \\
POD day 0 & $>30 \mathrm{mg} / \mathrm{g}(n=7)$ & $4(2-5)$ & $8.5(7-14)^{\mathrm{a}}$ \\
POD day 1 & 3rd tertile $(>75.8 \mathrm{mg} / \mathrm{g} ; n=17)$ & $5(3-5.5)^{\mathrm{a}}$ & $9(7.5-11.5)^{\mathrm{b}}$ \\
POD day 2 & 3rd tertile $(>80 \mathrm{mg} / \mathrm{g} ; n=16)$ & $4.5(3-5.75)^{\mathrm{a}}$ & $4(3-6)^{\mathrm{b}}$ \\
\hline
\end{tabular}

$I C U$, intensive care unit; $A C R$, albumin-to-creatinine ratio; $m g$, milligram; $g$, gram; $P O D$, post-operative day; $I Q R$, interquartile ${ }^{\mathrm{a}} p<0.05 ;{ }^{\mathrm{b}} p<0.001$ 
Table 6 Diagnostic characteristics of pre-operative and post-operative day 0 ACR threshold values to predict post-operative AKI

\begin{tabular}{|c|c|c|c|c|c|}
\hline & & Sensitivity & Specificity & PPV & NPV \\
\hline \multirow[t]{5}{*}{ Pre-operative } & $<2$ years & & & & \\
\hline & $\mathrm{ACR}>30 \mathrm{mg} / \mathrm{g}$ & 0.96 & 0.30 & 0.35 & 0.95 \\
\hline & $\begin{array}{l}\text { Maximal sensitivity and specificity value: } \geq 42.5 \mathrm{mg} / \mathrm{g} \\
\geq 2 \text { years }\end{array}$ & 0.77 & 0.61 & 0.43 & 0.87 \\
\hline & $\mathrm{ACR}>30 \mathrm{mg} / \mathrm{g}$ & 0.20 & 0.88 & 0.29 & 0.81 \\
\hline & Maximal sensitivity and specificity value: $\geq 14.5 \mathrm{mg} / \mathrm{g}$ & 0.80 & 0.48 & 0.28 & 0.90 \\
\hline \multirow[t]{7}{*}{ Post-operative at day 0} & $<2$ years & & & & \\
\hline & $>75 \mathrm{mg} / \mathrm{g}$ (2nd tertile or higher) & 0.88 & 0.40 & 0.37 & 0.90 \\
\hline & $>141.3 \mathrm{mg} / \mathrm{g}$ (3rd tertile of higher) & 0.54 & 0.75 & 0.45 & 0.81 \\
\hline & $\begin{array}{l}\text { Maximal sensitivity and specificity value: } \geq 119 \mathrm{mg} / \mathrm{g} \\
\geq 2 \text { years }\end{array}$ & 0.69 & 0.64 & 0.43 & 0.84 \\
\hline & $>35.9 \mathrm{mg} / \mathrm{g}$ ( $2 \mathrm{nd}$ tertile or higher) & 0.90 & 0.40 & 0.27 & 0.94 \\
\hline & $>75.8 \mathrm{mg} / \mathrm{g}$ (3rd tertile of higher) & 0.80 & 0.78 & 0.47 & 0.94 \\
\hline & Maximal sensitivity and specificity value: $\geq 72.5 \mathrm{mg} / \mathrm{g}$ & 0.90 & 0.73 & 0.45 & 0.97 \\
\hline
\end{tabular}

$A C R$, albumin-to-creatinine ratio; $m g$, milligram; $g$, gram; $P P V$, positive predictive value; $N P V$, negative predictive value

values were high while positive predictive values were low for different ACR threshold values (Table 6).

\section{Added benefit of pre- and first post-operative ACR to clinical predictors to predict AKI}

For the group aged $<2$ years, AUC $(95 \% \mathrm{CI})$ of pre-operative clinical model (0.761 (0.657-0.866)) for AKI prediction was relatively higher than pre-operative ACR alone (0.729 (0.618-0.840)), first post-operative ACR alone (0.704 (0.586-0.822)) and pre-operative ACR + first postoperative ACR (0.751 (0.645-0.856)). Similarly, for the group aged $\geq 2$ years, AUC $(95 \% \mathrm{CI})$ of pre-operative clinical model (0.842 (0.716-0.969)) was relatively higher than pre-operative ACR alone (0.639 (0.456-0.821)), first post-operative ACR alone (0.803 (0.618-0.987)) and pre-operative ACR + first post-operative ACR (0.688 (0.506-0.869)). A relatively higher increase in area under the ROC curve was observed by adding pre-operative ACR ( $<2$ years: $0.805(0.713-0.896) ; \geq 2$ years: 0.872 $(0.772-0.973))$ than post-operative ACR $(<2$ years: 0.777 $(0.676-0.879)$; $\geq 2$ years: $0.844(0.718-0.969))$ to the clinical model. Also, it was observed that the area under the ROC curve for clinical model + pre-operative ACR $(<2$ years: $0.805(0.713-0.896) ; \geq 2$ years: $0.872(0.772-0.973)$ ) was slightly higher than that for clinical model + pre-operative ACR + first post-operative ACR $(<2$ years: 0.799 (0.705-0.893); $\geq 2$ years: $0.862(0.751-0.974))$, indicating no incremental value of adding post-operative ACR in predicting AKI (Table 7).

Table 7 Improvement in AUC as a result of adding pre-operative and first post-operative ACR to clinical predictive models

\begin{tabular}{|c|c|c|c|c|}
\hline \multirow[t]{2}{*}{ Model } & \multicolumn{2}{|l|}{$<2$ years } & \multicolumn{2}{|l|}{$\geq 2$ years } \\
\hline & $\mathrm{AUC}(95 \% \mathrm{CI})$ & $p$ value & AUC (95\% CI) & $p$ value \\
\hline Clinical model ${ }^{\wedge}$ alone & $0.761(0.657-0.866)$ & $<0.001$ & $0.842(0.716-0.969)$ & 0.001 \\
\hline Pre-operative ACR alone & $0.729(0.618-0.840)$ & 0.001 & $0.639(0.456-0.821)$ & 0.178 \\
\hline First post-operative ACR alone & $0.704(0.586-0.822)$ & 0.002 & $0.803(0.618-0.987)$ & 0.003 \\
\hline Pre-operative ACR + first post-operative ACR & $0.751(0.645-0.856)$ & $<0.001$ & $0.688(0.506-0.869)$ & 0.049 \\
\hline Clinical model^ + pre-operative ACR & $0.805(0.713-0.896)$ & $<0.001$ & $0.872(0.772-0.973)$ & $<0.001$ \\
\hline Clinical model ${ }^{\wedge}+$ first post-operative ACR & $0.777(0.676-0.879)$ & 0.001 & $0.844(0.718-0.969)$ & 0.001 \\
\hline $\begin{array}{l}\text { Clinical model }{ }^{\wedge}+\text { Pre-operative ACR }+ \text { first post- } \\
\text { operative ACR }\end{array}$ & $0.799(0.705-0.893)$ & $<0.001$ & $0.862(0.751-0.974)$ & $<0.001$ \\
\hline
\end{tabular}

$A U C$, area under the curve; $C I$, confidence Interval; $A C R$, albumin-to-creatinine ratio; $G F R$, glomerular filtration rate; $R A C H S$, risk adjustment in congenital heart surgery

${ }^{\wedge}$ The clinical predictive model includes age (continuous in the group aged $<2$ years; categorized as $2-<6$ versus $\geq 6$ years in the group aged $\geq 2$ years), gender, RACHS-1 score $\geq 3$ versus $<3$, creatinine (continuous), GFR (continuous), type of heart surgery (categorical), previous cardiac surgeries (categorical) and cardiopulmonary bypass time ( $<120$ versus $\geq 120 \mathrm{~min}$ ) 


\section{Discussion}

This study identifies that prediction of developing AKI in children post cardiac surgery using cardiopulmonary bypass increases with use of the pre-operative. The clinical model had the highest AUC in comparison to pre-operative and/or first post-operative ACR alone in predicting AKI. A combination of clinical model and - pre-operative ACR produced the best performance in AKI prediction, providing for a collective assessment of AKI risk in children undergoing open cardiac surgery. An early evidence of AKI can help in change of practices which may exacerbate kidney dysfunction, such as use of nephrotoxic drugs like aminoglycosides or NSAIDs, judicious use of contrast, optimisation of volume status and allow for prognostication and timely interventions.

Scores based on clinical variables have been previously used and validated for assessing AKI risk in settings of cardiac surgery [20,21]. As discussed by Tinica and colleagues in a systematic review of AKI determinants after cardiac surgery in adults, most clinical scores are often able to identify severe forms of AKI but may miss mild cases and incorporation of biomarkers such as proteinuria among others would help in improving AKI risk ascertainment [22]. Zappitelli et al. in a similar study assessing albuminuria in a paediatric population following open cardiac surgery also identified a better AKI prediction model when utilising clinical variables, first post-operative ACR and cystatin C [14]. Koyner et al. were also able to demonstrate a greater determination of AKI severity progression post cardiac surgery in adults with use of biomarkers such as plasma NGAL, urine ACR and urine IL-18 over risk scores. Plasma NGAL performed best followed by urine ACR (odds ratio of 3.4) [23].

The median albuminuria in age group $<2$ years with AKI was also significantly higher at all time points (preand post-operatively) in comparison to those without AKI, as opposed to children $\geq 2$ years with AKI only showing a significant difference in the first early post-operative ACR. However, the pre-operative ACR was unable to predict AKI in adjusted analyses for either age group. Incorporation of clinical model to preoperative ACR had the highest AUC for prediction of AKI. Similar findings have been seen in adults wherein pre-operative proteinuria has shown significant association with subsequent AKI following both cardiac and non-cardiac surgeries [24-26], with risk increasing in tandem with greater levels of proteinuria. As comorbidities such as diabetes mellitus, hypertension and their complications tend to accrue with age, higher pre-operative proteinuria in adults with subclinical kidney impairment is plausible. In children, it is known that congenital heart diseases may accompany kidney abnormalities. Moreover, it is known that presence of congenital heart disease leads to altered physiology states, hemodynamic and hypoxic insults which can be the cause of pre-existing renal impairment in this vulnerable population [24].

Post-operatively, ACR rose in both age groups irrespective of development of AKI, possibly as a result of inflammatory or hemodynamic stress. In children $>2$ years, the highest tertile $(>75.8 \mathrm{mg} / \mathrm{g})$ of the first post-operative ACR was also able to predict AKI even after adjusting for clinical parameters (adjusted RR 11.71; CI 1.85-16.59); in children under 2 years $(>141.3 \mathrm{mg} / \mathrm{g})$, it was able to do so in unadjusted analyses but not after adjusting for clinical factors.

In contrast, in the study conducted by Zappitelli and colleagues, the highest tertile of first post-operative ACR was able to predict stage 1 and 2 AKI even after controlling for clinical variables in all age groups [14]. Devarajan et al. also performed urinary proteomic analysis in children post cardiac surgery to look for putative biomarkers. They found three urinary protein peaks, corresponding to $\alpha 1$-microglobulin, $\alpha 1$-acid glycoprotein and albumin, to be significantly increased in children who developed AKI (increase $2 \mathrm{~h}$ post-surgery from baseline and checked at various time points up to $72 \mathrm{~h}$ ); these findings were confirmed in a validation set [27].

Increased ACR levels also were significantly associated with longer ICU and hospital stays in all age groups, which signifies that kidney injury even when subtle can increase morbidity and affect outcomes adversely. Due to low event numbers (3), the data on mortality was not definitive. Devarajan and colleagues also found increased duration of hospital stay and AKI with increasing quartiles of three urinary biomarkers (including albumin) post open cardiac surgery in children [27].

In comparison with the Zappitelli et al. study, our study highlights the importance of adding pre-operative rather than post-operative ACR to the clinical model in predicting AKI, which is also reported in adults [13]. Our study also demonstrates that there is no incremental value of adding post-operative ACR for AKI prediction, while Zappitelli et al. found no improvement by incorporating pre-operative ACR in first post-operative ACR performance in any of the models. Additionally, the AUCs reported in our study are relatively higher but within the $95 \%$ CI interval reported by Zappitelli et al. For the clinical predictive model, some additional variables were considered in our study, such as previous cardiac surgeries, type of heart surgery and pre-operative creatinine as compared to that by Zappitelli et al. However, other serum or urinary biomarkers were not considered for the analysis as was done by Zappitelli et al. [13, 15].

The strengths of the present study are its prospective nature and standardised laboratory assessments of albuminuria. Our study had a few limitations including low number 
of events especially in older children. Being a single centre study, it affects the generalisability of its results, especially in non-cardiac settings. We did not use other serum or urinary biomarkers in our analysis which could have provided greater information.

\section{Conclusion}

This study has enhanced the use of albuminuria in AKI prediction. Preoperative ACR when added to a clinical model helps predict AKI in all age groups. It allows for AKI risk evaluation, allowing for initiation of preventive measures, better monitoring and prognostication and thus better management of children undergoing open cardiac surgery. It provides evidence for use of albuminuria as a feasible biomarker in AKI and studies assessing the same in different risk populations could provide further corroborating evidence.

Abbreviations AKI: Acute kidney injury; RAI: Renal angina index; ACR: Urinary albumin:creatinine ratio; KDIGO: Kidney Disease Improving Global Outcomes; CPB: Cardiopulmonary bypass; RACHS1: Risk adjustment for congenital heart surgery; AUC: Area under the curve; POD: Post-operative day; NGAL: Neutrophil gelatinase-associated lipocalin

Supplementary Information The online version contains supplementary material available at https://doi.org/10.1007/s00467-021-05219-0.

\section{Declarations}

Competing interests The authors declare no competing interests.

\section{References}

1. Li S, Krawczeski CD, Zappitelli M, Devarajan P, ThiessenPhilbrook H, Coca SG, Kim RW, Parikh CR (2011) Incidence, risk factors, and outcomes of acute kidney injury after pediatric cardiac surgery - a prospective multicenter study. Crit Care Med 39:1493-1499

2. Mao H, Katz N, Ariyanon W, Blanca-Martos L, Adýbelli Z, Giuliani A (2013) Cardiac surgery associated acute kidney injury. Cardiorenal Med 3:178-199

3. Zappitelli M, Bernier P-L, Saczkowski RS, Tchervenkov CI, Gottesman R, Dancea A (2009) A small post-operative rise in serum creatinine predicts acute kidney injury in children undergoing cardiac surgery. Kidney Int 76:885-892

4. Alam S, Shalini A, Hegde R, Mazahir R (2017) Acute kidney injury as a predictor of poor outcome post cardiopulmonary bypass in children. Int Contemp Pediatr 4:234-240

5. Ostermann M, Zarbock A, Goldstein S, Kashani K, Macedo E, Murugan R, Bell M, Forni L, Guzzi L, Joannidis M, KaneGill SL, Legrand M, Mehta R, Murray PT, Pickkers P, Plebani M, Prowle J, Ricci Z, Rimmelé T, Rosner M, Shaw AD, Kellum JA, Ronco C (2020) Recommendations on acute kidney injury biomarkers from the Acute Disease Quality Initiative
Consensus Conference: a consensus statement. JAMA Netw Open 3:e2019209. https://doi.org/10.1001/jamanetworkopen. 2020.19209

6. Menon S, Goldstein SL, Mottes T, Fei L, Kaddourah A, Terrell T, Arnold P, Bennett MR, Basu RK (2016) Urinary biomarker incorporation into the renal angina index early in intensive care unit admission optimizes acute kidney injury prediction in critically ill children: a prospective cohort study. Nephrol Dial Transplant 31:586-594

7. Meersch M, Schmidt C, Hoffmeier A, Van Aken H, Wempe C, Gerss J, Zarbock A (2017) Prevention of cardiac surgery-associated AKI by implementing the KDIGO guidelines in high risk patients identified by biomarkers: the PrevAKI randomized controlled trial. Intensive Care Med 43:1551-1561

8. Levey AS, de Jong PE, Coresh J, El Nahas M, Astor BC, Matsushita K, Gansevoort RT, Kasiske BL, Eckardt KU (2011) The definition, classification, and prognosis of chronic kidney disease: a KDIGO Controversies Conference report. Kidney Int 80:17-28

9. Ware LB, Johnson AC, Zager RA (2011) Renal cortical albumin gene induction and urinary albumin excretion in response to acute kidney injury. Am J Physiol Renal Physiol 300:F628-638

10. Parr SK, Matheny ME, Abdel-Kader K, Greevy RA Jr, Bian A, Fly J, Chen G, Speroff T, Hung AM, Ikizler TA, Siew ED (2018) Acute kidney injury is a risk factor for subsequent proteinuria. Kidney Int 93:460-469

11. Yu Y, Jin H, Holder D, Ozer JS, Villarreal S, Shughrue P, Shi S, Figueroa DJ, Clouse H, Su M, Muniappa N, Troth SP, Bailey W, Seng J, Aslamkhan AG, Thudium D, Sistare FD, Gerhold DL (2010) Urinary biomarkers trefoil factor 3 and albumin enable early detection of kidney tubular injury. Nat Biotechnol 28:470-477

12. Coca SG, Jammalamadaka D, Sint K, Thiessen Philbrook H, Shlipak MG, Zappitelli M, Devarajan P, Hashim S, Garg AX, Parikh CR, Translational Research Investigating Biomarker Endpoints in Acute Kidney Injury Consortium (2012) Preoperative proteinuria predicts acute kidney injury in patients undergoing cardiac surgery. J Thorac Cardiovasc Surg 143:495-502

13. Huang TM, Wu VC, Young GH, Lin YF, Shiao CC, Wu PC, Li WY, Yu HY, Hu FC, Lin JW, Chen YS, Lin YH, Wang SS, Hsu RB, Chang FC, Chou NK, Chu TS, Yeh YC, Tsai PR, Huang JW, Lin SL, Chen YM, Ko WJ, Wu KD (2011) National Taiwan University Hospital Study Group of Acute Renal Failure. Preoperative proteinuria predicts adverse renal outcomes after coronary artery bypass grafting. J Am Soc Nephrol 22:156-163

14. Zappitelli M, Coca SG, Garg AX, Krawczeski CD, ThiessenPhilbrook HT, Sint K, Li S, Parikh CR, Devarajan P (2012) The association of albumin/creatinine ratio with postoperative AKI in children undergoing cardiac surgery. Clin J Am Soc Nephrol 7:1761-1769

15. Kidney Diseases Improving Global Outcomes (2012) KDIGO clinical practice guideline for acute kidney injury. Kidney Int 2(Suppl 1):1-138

16. Schwartz GJ, Muñoz A, Schneider MF, Mak RH, Kaskel F, Warady BA, Furth SL (2009) New equations to estimate GFR in children with CKD. J Am Soc Nephrol 20:629-637

17. Jenkins KJ, Gauvreau K, Newburger JW, Spray TL, Moller JH, Iezzoni LI (2002) Consensus-based method for risk adjustment for surgery for congenital heart disease. J Thorac Cardiovasc Surg 123:110-118

18. Goldstein SL, Currier H, Graf JM, Cosio CC, Brewer ED, Sachdeva R (2007) Outcome in children receiving continuous venovenous hemofiltration. Pediatrics 107:1309-1312

19. Hoffman TM, Wernovsky G, Atz AM, Bailey JM, Akbary A, Kocsis JF, Nelson DP, Chang AC, Kulik TJ, Spray TI, Wessel DL (2002) Prophylactic intravenous use of milrinone after cardiac operation in pediatrics (PRIMACORP) study. Prophylactic 
Intravenous Use of Milrinone After Cardiac Operation in Pediatrics. Am Heart J 143:15-21

20. Thakar CV, Arrigain S, Worley S, Yared J-P, Paganini EP (2005) A clinical score to predict acute renal failure after cardiac surgery. J Am Soc Nephrol 16:162-168

21. Mehta RH, Grab JD, O'Brien SM, Bridges CR, Gammie JS, Haan CK, Ferguson TB, Peterson ED; Society of Thoracic Surgeons National Cardiac Surgery Database Investigators (2006) Bedside tool for predicting the risk of postoperative dialysis in patients undergoing cardiac surgery. Circulation 114:2208-2216

22. Tinica G, Brinza C, Covic A, Popa IV, Tarus A, Bacusca AE, Burlacu A (2020) Determinants of acute kidney injury after cardiac surgery: a systematic review. Rev Cardiovasc Med 21:601-610

23. Koyner JL, Garg AX, Coca SG, Sint K, Thiessen-Philbrook H, Patel UD, Shlipak MG, Parikh CR; TRIBE-AKI Consortium (2012) Biomarkers predict progression of acute kidney injury after cardiac surgery. J Am Soc Nephrol 23:905-914

24. Zheng J, Yao Y, Han L, Xiao Y (2013) Renal function and injury in infants and young children with congenital heart disease. Pediatr Nephrol 28:99-104
25. Wahl TS, Graham LA, Morris MS, Richman JS, Hollis RH, Jones CE, Itani KM, Wagner TD, Mull HJ, Whittle JC, Telford GL, Rosen AK, Copeland LA, Burns EA, Hawn MT (2018) Association between preoperative proteinuria and postoperative acute kidney injury and readmission. JAMA Surg 153:e182009

26. Park S, Lee S, Lee A, Paek JH, Chin HJ, Na KY, Chae DW, Kim S (2018) Preoperative dipstick albuminuria and other urine abnormalities predict acute kidney injury and patient outcomes. Surgery 163:1178-1185

27. Devarajan P, Krawczeski CD, Nguyen MT, Kathman T, Wang Z, Parikh CR (2010) Proteomic identification of early biomarkers of acute kidney injury after cardiac surgery in children. Am J Kidney Dis 56:632-642

Publisher's note Springer Nature remains neutral with regard to jurisdictional claims in published maps and institutional affiliations. 